\title{
Reviewing Anemia and Iron Folic Acid Supplementation Program in Bangladesh - A Special Article
}

\author{
M Rashid1, M S Flora2, M A Moni3, A Akhter4, Z Mahmud5
}

\begin{abstract}
Despite supplementation of iron folic acid tablets over the past few decades, no marked improvement has been noticed in the magnitude of anemia in Bangladesh. It has been a felt need to have a fresh look at the problem of iron/folic acid deficiency anemia prevailing in Bangladesh and the perspective of the supplementation program. Data were collected through a review of a good number of available documents and in-depth interviews of concerned policy makers, stakeholders and care
\end{abstract}

\section{Key Words : Anemia, Bangladesh, IFA supplementation Introduction}

Anemia is a widespread public health problem in Bangladesh. It poses a major threat to maternal and child survival, contributes to low birth weight, lowered resistance to infection, poor cognitive development and decreased work productivity. It has profound effect on health, both physical and mental, thereby affecting quality of life, and translating into significant economic losses for the individuals and for the country. These impacts are estimated to cost Bangladesh $7.9 \%$ of its gross domestic product (GDP).

Anemia has multiple factors, many of which can occur simultaneously in the same individual. The contribution of each cause of anemia to the high prevalence in Bangladesh cannot be determined from the data available. However, it is highly likely that iron deficiency is the single most important cause, as observed in most countries of the world.

Healthy normal weight newborns usually have adequate iron stores at birth to last them until about six months of life, provided they are exclusively providers. An iron folic acid supplementation component is formally in place in the Health, Nutrition, and Population Sector Program (HNPSP) and implemented by both the health and family planning wings of the Ministry of Health and Family Welfare (MoHFW). The iron folic acid component primarily focuses on the pregnant and lactating women and fails to focus other groups who also are equally affected. Tablets are provided on a weekly basis, thus not completing the required dose. A holistic approach is required to combat this long standing problem.

breastfed. However, 36\% of infants (some studies show higher figures) in Bangladesh are born with low birth weight and with low body iron stores that are quickly exhausted in the first few months of life. Furthermore, only $42 \%$ of Bangladeshi infants are exclusively breastfed for six months and the quality, quantity and frequency of complementary feeding is inadequate to meet their requirements from six months onwards.

Urban preschool children have higher anemia prevalence than their rural counterparts. Anemia is more common among the poor. Among mothers and household members, there is a lack of knowledge on dietary practices that could protect family members from irondeficiency. They also lack knowledge on how to make their diet more nutritious and on appropriate cooking methods.

Within urban areas, the prevalence of anemia is higher in the slum areas compared to that of the non-slum areas. Rural areas of the Chittagong Hill tracts (CHT) have higher anemia prevalence than elsewhere in rural Bangladesh and in the urban areas of the country.

The magnitude of anemia and its adverse health and economic consequences connote the need for intensified action to address this public health problem. In 2007, the Bangladesh government adopted a "National Strategy for the Prevention and Control of Anemia". The current document is an approach to analyze this problem over a period of time, the measures that have already been taken, and the scope of future works to prevent and control this devastating issue. 


\section{Methodology}

In order to carry out the review, several evaluation reports available with different departments of the government, national institutes and international organizations operating in Bangladesh were looked into. The current government procurement and logistics system, training components of health care providers, existing status of the MIS of both the Health and Family Planning wings of the government were carefully examined by interviewing a number of key personnel. Face to face in-depth interviews were carried out for this purpose and comments were invited through these direct individual meetings. Although a common format of an interview schedule was developed, it was used only as a guideline and modified accordingly, depending on who was being interviewed and what his expertise/job was. Some people were also interviewed at the district and upazilla level to gather on hand information as found in the field. A trend analysis of anemia and iron deficiency over the last 10 years in different population sub-groups in the country was tried based on the available evidence and literature. With background information coming from the secondary data, the rest of the report is based mostly on that obtained from the stake holders.

\section{Findings}

Anemia situation in Bangladesh

According to international criteria, anemia is a severe public health concern (defined as a prevalence of $40 \%$ or more) in preschool age children and pregnant women, and a moderate problem (defined as prevalence between 20-39\%) in adolescent girls and non-pregnant women of reproductive age. According to BBS/UNICEF (2004), anemia affects $46 \%$ of pregnant women, $64 \%$ of children aged $6-23$ months, $42 \%$ of children aged 24-59 months, 30\% of adolescent girls and $33 \%$ of non-pregnant women. The prevalence of anemia is particularly high among children aged 6-23 months and pregnant women because their nutritional requirements for growth and reproduction are relatively much higher. The anemia prevalence survey also included urban samples which indicated marked anemia; however prevalence was lower than rural samples. The prevalence of anemia in urban areas were $56 \%$ in $<5$ year old children, $29 \%$ and $17 \%$ in adolescent girls and boys, respectively. In addition, $41 \%$ of pregnant and $33 \%$ of non-pregnant women were found to be anemic.

National level prevalence data for the last 10 years were looked into. No recent data in this regard could be found. Findings from a survey conducted in rural Bangladesh by the Nutritional Surveillance Project (NSP) of Helen Keller International (HKI) in collaboration with the Institute of Public Health Nutrition (IPHN) in 2001 showed that $48 \%$ of $<5$ year old children were anemic. Approximately $30 \%$ of the adolescent girls and $29 \%$ of the adolescent boys suffered from anemia as well as $47 \%$ of the pregnant and $34 \%$ of the non-pregnant women. The anemia prevalence survey by the Bangladesh Bureau of Statistics (BBS) under the Monitoring Situation of Children and Women (MSCW) Project in collaboration with UNICEF and BRAC in 2003 revealed that the prevalence of anemia was higher than the 2001 NSP survey. The Nutritional Surveillance Project (NSP) of HKI repeated a similar survey in 2004 and the

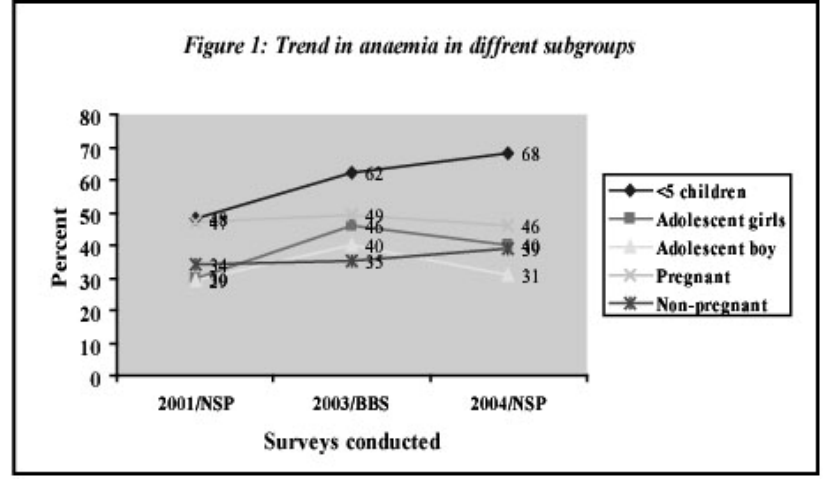

A trend in childhood anemia in Bangladesh was constructed with data from national surveys with varying sample sizes among children 6-69.9 months execept the 2009 data which was conducted among the under 2 children in nor thern char areas of Bangladesh (Fig. -2.)In 1997, the

Figure 2: Trends in anaemia among children

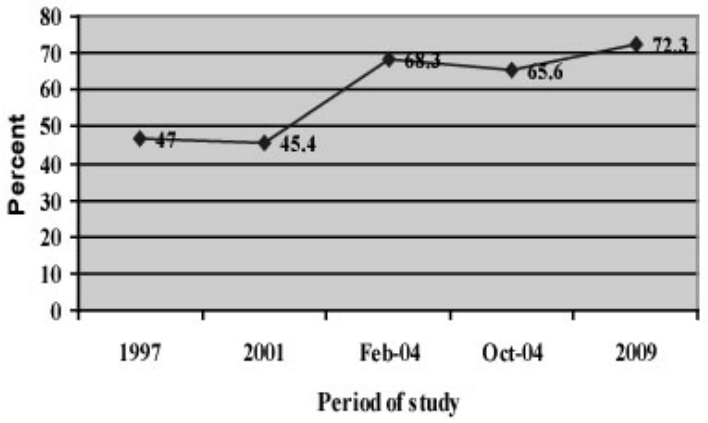


data were collected from the National Vitamin A Deficiency (VAD) survey, while the 2001 and 2004 surveys were done under the Nutritional Surveillance Project (NSP). Ignoring the minor differences thatresult showed a gradual increase in the prevalence of anemia in $<5$ year children but a decrease was observed in other groups as compared to the BBS 2003 survey. These differences might be attributed to the methodological differences in the two surveys (Figure 1 ).

children 6-59.9 months except the 2009 data which was conducted among the under 2 children in the northern char areas of Bangladesh (Figure 2).

could arise due to adoption of different methodologies, it can be clearly stated that anemia in children is highly prevalent in Bangladesh and within the last 10 years it has increased markedly. Although from limitedsamples, the recent data suggest a marked increase in prevalence which is alarming.

\section{Anemia control activities in Bangladesh}

The commitment of the government in addressing anemia is seen through the Poverty Reduction Strategy Paper and the Health, Nutrition and Population Sector Program for 2003-10, in which the prevention and control of anemia is one of the key strategies for reducing maternal, neonatal and childhood mortality and improving maternal and childhood nutrition. Anemia prevention and control also features in the 1997 National Plan of Action for Nutrition (NPAN) and the National Plan of Action for Children (2004-2009). The MoHFW has overall responsibilities for all activities related to anemia control in Bangladesh through the Directorate General of Health Services (DGHS), Directorate General of Family Planning (DGHS) and the National Nutrition Programme (NNP).

In 2001, the Institute of Public Health Nutrition developed National Guidelines for the Prevention and Treatment of Iron Deficiency Anemia, which recommend iron supplementation, dietary improvement, food fortification and helminth control in preschool-aged children, school-aged children, adolescent girls, and women of reproductive age.

\section{The National Strategy}

The overall goal of the National Strategy is to reduce by one quarter the prevalence of anemia among the high-risk groups in Bangladesh by 2015.

Target Groups: Interventions are prioritized to the three high risk groups at greatest risk of anemia and its consequences including low birth weight infants aged 2-5 months and all children aged 6-23 months, pregnant women and breastfeeding women up to 3 months postpartum, and adolescent girls and newly married women. Subject to availability of resources, three additional groups are also to be considered. They are pre-school children aged 24-59 months, school-aged children aged 5-11 years, non-pregnant women of reproductive age.

Strategies: Strategies fall into two categories- population based strategies and targeted strategies for high-risk groups. Population-based strategies include food fortification, production of micronutrient-rich foods through household food production and crop diversification, biotechnology and biofortification. Targeted strategies for high-risk groups include micronutrient supplementation, dietary improvement, parasitic disease control and, family planning and safe motherhood.

\section{Anemia control measures for specific groups}

For women: In most areas of the country, the only strategy to prevent and control anemia has been the iron-folate supplementation (IFA) during pregnancy with daily supplements of $60 \mathrm{mg}$ of iron and $400 \mathrm{ug}$ folate from the second trimester onwards till 42 days after delivery by the Family Welfare Visitors of the DGFP as part of the antenatal care services at Satellite Clinics and Maternal and Child Welfare Centers (MCWC). DGHS provides iron-folic acid supplements to pregnant women through its reproductive health programme during antenatal care at different levels of health care services. In addition to supplying IFA to the pregnant and lactating women, the NNP promotes dietary practices among women through the Community Nutrition Promoters (CNP). Several national and international NGOs and the private sector also provide IFA supplements to pregnant and breastfeeding women, both in the rural and urban areas of Bangladesh.

For Adolescents: Adolescent girls are not included in nationwide efforts. A program is running for the last five years for girl students of class 7 - 10. One iron folic acid capsule per week is distributed to those students of government and nongovernment schools of areas outside Dhaka city. Primary school 
functioning, are trained on anemia prevention targeting awareness creation among the primary school students through them. No survey has yet been carried out on the status of anemia before and after running this program. The DGFP also offers IFA for the adolescents as part of the RTI/STI program. In its operational area, the NNP has taken a broader approach to anemia control by also including adolescent girls, and by providing anthelminthic treatment to them. Importantly, these interventions are provided at the community level by the CNPs. The NNP also promotes dietary practices among adolescent girls through the same CNPs.

For Children: Nationwide, children aged less than five years are not routinely given any intervention to prevent and control anemia. IMCI is addressing malnutrition along with NNP. The NNP also promotes breastfeeding, complementary feeding through the CNPs. Deworming of children aged 24-59 months with albendazole is included during the six-monthly National Vitamin A Plus Campaigns (NVAC).

UNICEF is involved mainly in policy/strategy development. To combat anemia, through their involvement, children in some of the Dhaka urban slums and CHT are receiving multiple micronutrient powder which contains 5 nutrients including iron and folic acid. The WHO formulation of $60 \mathrm{mg}$ elemental iron is followed. For childhood anemia, the DGFP and UNICEF are at a planning stage to develop a program for them. DGPF, ICDDR, B and UNICEF are planning to launch a large program to supply micronutrient powder to 6-23 month children.

Receipt and compliance of supplements by target beneficiaries Those who are suffering from anemia do not take it seriously and often accept it as a normal phenomenon or at best a very minor ailment not leading to a serious or bed-ridden condition. Sometimes health care providers are advised to supply only six tablets on a single visit which is to be refilled only if they return. In many cases the clients do not return and treatment remains incomplete. In the Medical Colleges it is seen that tablets are prescribed for 100 days but the supply is found that only $15 \%$ of rural pregnant women take at least 100 IFA tablets during pregnancy and $46 \%$ take none. The access to this supplement is also limited as only $27 \%$ of women make 3 or more antenatal care visits during pregnancy.

\section{Procurement and Distribution of Iron Folate Tablets} The Line Director (LD) of NNP (the ED) and LD of DGHS (PHC) are required to place their requirements to the LD of CMSD (the central medical store). After receiving the demand note, CMSD goes for National or International Competitive Bidding. If the price goes above Taka 2 crores, they have to go for International Competitive Bidding (ICB).

For the NNP, the demand for Iron and Folic Acid comes from the NGOs. The Public Procurement Regulations are followed in buying Iron and Folic Acid Tablets. Roughly, around 35 crore tablets are needed per year which comes to about 2 crore 70 lacs tablets for each of the upazillas where NNP is in place. For the DGHS, the procurement of IFA is done through the SLPP. Basically the amount procured last year forms the basis of need calculation for the current year.

For the DGFP, all drugs are supplied in the DDS kits and each kit contains 2000 IFA tablets. One lac ten thousand DDS kits are supplied each year. IFA is obtained from the EDCL (essential drugs company). Local tender is made from the revenue budget. It is stored in the Mohakhali Central Ware House belonging to the DGFP. From there it goes to the District Reserve Stores (DRS) and finally to some 250 upazilla stores and ultimately to the field.

Community Clinics (CC) receive IFA tablets from the UHFPO. IFA tablets for the CC are mainly produced by EDCL. The tablets at first come to the DRS and are then sent to the UHC. Tablets are supplied according to allotment.

\section{Training of health care provider}

Anemia as a topic is widely covered in the different courses conducted for the undergraduate and post graduate medical courses. Occasional seminars are held for the experts and nutritional disorders are discussed. Topic related to anemia is covered in the
Bangladesh Medical Journal 2010 Vol. 39 No. 3

IMCI training. The FWVs get 18 months of basic training. These trainings continue in phases and nutrition including

\section{September 2010 Issue}

is no shortage of IFA supplies at the local level might be a fallacy. If the full course of the required IFA is provided, then 
anemia is often a component. The FWAs receive a similar training for 1 month. The SBAs also receive training on nutrition in their 6 month long training program.

\section{Advocacy and Behavior Change Communication}

Posters (pictures) and other materials have been developed by the government, donor agencies and NGOs. The media remains under utilized as far as promotion activities to tackle anemia is concerned.

\section{Monitoring Information Systems}

DGHS: Disease profile data by age and sex from OPD and indoor patients come to MIS. Anemia is one of the reported top ten diseases. Unfortunately, there is neither data on IFA consumption or compliance.

DGFP: The MIS forms are filled in by the ANC providers which ultimately reaches the head office. It could not be verified if data is being electronically transferred because it was reported that all data/information still reaches the head quarter at Kawran Bazar in Dhaka by the postal service.

\section{Discussion}

Education and motivation will play a vital role in handling anemia. The ANC services should be improved, and efforts should be made so that awareness is created not only amongst the sufferers but also in those who are entrusted with to mitigate the problem. Health service providers are either not aware or are not applying the IPHN guidelines for the prevention and control of anemia. They lack knowledge, counseling skills, communication materials, time and motivation to counsel mothers on the importance of IFA and other interventions to prevent anemia and how to manage sideeffects, and to monitor compliance with IFA supplementation. As a study suggests, there is a clear need to improve awareness on anemia and the need for prevention, as common reasons cited by women for not taking supplements are that "they are not necessary" (49\%), "no-one told me to take them/I did not know I needed them" (15\%) and "side-effects" (13\%). The health workers should be encouraged to provide enough IFA supplements to last until the next ANC visit. The notion that there the amount of IFA tablets may not be sufficient. IFA supplements are uncoated and wrapped in paper, which in humid climates does not protect the tablets from spoilage. The tablets can be provided in small containers with lids so that the tablets are not wasted in a short span of time.

IFA supplementation does not seem to be the responsibility of any one in particular. There is a lack of coordination in providing ANC as the role of DGFP or PHC is not clearly defined. NGOs are also not involved in the main stream of service delivery and there seems to be a lack of coordination in IFA distribution between the government departments and the NGOs which might affect the optimal utilization of resources. Here a scope lies for the Institute of Public Health Nutrition (IPHN) to play a role in coordinating the anemia control and IFA supplementation issue. A coordinated and comprehensive approach to anemia prevention and control is needed in Bangladesh. IPHN could play a pivotal role through strategy and policy making, linking and coordinating between different departments and sectors concerned and in the procurement and distribution of IFA supplements.

At certain time intervals, national level anemia surveys among all the different risk groups are very crucial to understand the current situation. Research on this great public health issue needs to be strengthened.

\section{Conclusion}

Despite IFA supplementation being in place for many years, the magnitude of anemia is still high. A holistic approach to control anemia emphasizing on training of the workers, community awareness, expansion of supplementation areas and population targeting is recommended. Evidence-based approaches would result in successful anemia control.

\section{Acknowledgement}

This review was based on an assignment done with the support and cooperation of Canadian based Micronutrient Initiatives (MI), working in Bangladesh. The authors are grateful to all the
Bangladesh Medical Journal 2010 Vol. 39 No. 3

stakeholders starting from high level policy makers, international agencies and health care providers who participated in this study.
September 2010 Issue

9. IPHN. National Guidelines: Prevention and Treatment of Iron Deficiency Anaemia. IPHN, Dhaka, Bangladesh: 2001. 10. Jahan K. and Hossain M. Nature and extent of malnutrition 


\section{References}

1. BBS/UNICEF. Anemia prevalence survey of Urban Bangladesh and Rural Chittagong Hill Tracts 2003. Dhaka: 2004.

2. BBS. Bangladesh Health and Demographic Survey 2004. Dhaka: 2004.

3. Behrman J.R., Alderman H. \& Hoddinott, J. Nutrition and Hunger. In: Global Crises, Global Solutions (ed. Bjorn Lomborg). Cambridge University Press, Cambridge, UK: 2004.

4. Haseen F. Efficacy of daily and weekly home fortification of weaning foods with Sprinkles in improving iron deficiency anaemia among young children in Bangladesh. BRAC, Dhaka: 2004.

5. Haseen F. Hyder, Z., Ip, H., Rahman, M. \& Zlotkin, S. Trial Effectiveness of Daily and Flexible Administration of Micronutrient Sprinkles to Control Anaemia in Young Children in Rural Bangladesh, BRAC, Dhaka: 2005.

6. Government of Bangladesh. Unlocking the potential: National Strategy for Accelerated Poverty Reduction. General Economics Division, Planning Commission, government of Bangladesh, Dhaka: 2005.

7. HKI/IPHN. Anaemia is a serious public health problem in pre-school children and pregnant women in rural Bangladesh. NSP Bull. No.10. March 2002 HKI/IPHN, Dhaka: 2002.

8. HKI/IPHN. The burden of anaemia in rural Bangladesh: An urgent need for action. NSP Bull. No.16. April 2006. HKI/IPHN, Dhaka: 2006. in Bangladesh. Bangladesh National Nutrition Survey, 199596. Institute of Nutrition and Food Science, University of Dhaka, Dhaka, Bangladesh: 1998.

11. Lutter C.K. and Rivera J.A. Nutritional Status of Infants and Young Children and Characteristics of $\mathrm{f}$ Their Diets. Journal of nutrition 2003; 133: S2941-S2949.

12. NNP/UNICEF. Baseline knowledge, attitudes and practices survey of the National Nutrition Programme. NNP and UNICEF, Dhaka: 2004.

13. NPAN. National Plan of Action for Nutrition (1997), Ministry of Health and Family Welfare, government of the people's Republic of Bangladesh in Collaboration with Bangladesh National Nutrition Council. Dhaka:1997

14. United Nations System Standing Committee on Nutrition (SCN). 5th Report on the World Nutrition. Situation: Nutrition for improved development outcomes, SCN, March 2004, p. 14. 15. WHO/WFP/UNICEF. Joint statement by the World Health Organization, the World Food Programme: and the United Nations Children's Fund. Preventing and controlling micronutrient deficiencies in populations affected by emergency situations. Multiple Vitamin and Mineral Supplements for pregnant and lactating women and for children aged 6 to 59 months.2005. 